\title{
A COVID-19 pneumonia case report of autoimmune polyendocrine syndrome type 1 in Lombardy, Italy: letter to the editor
}

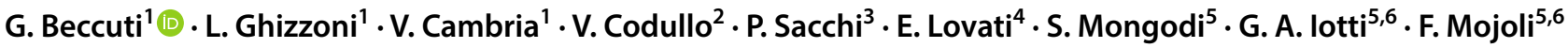

Received: 20 May 2020 / Accepted: 1 June 2020 / Published online: 9 June 2020

(C) Italian Society of Endocrinology (SIE) 2020

We thank the European Society of Endocrinology (ESE) for providing the global endocrinology community with the statement on coronavirus disease (COVID-19) and endocrine disorders [1]. Regarding Addison's disease, the ESE statement affirms that there is no evidence that patients with adrenal insufficiency are at increased risk of contracting COVID-19, and there are no reported data on the outcomes of COVID-19 infection in adrenal-insufficient individuals. Herein, we present the case of a 32-year-old woman with autoimmune polyglandular syndrome type 1 who developed COVID-19 caused by severe acute respiratory syndrome coronavirus 2 (SARS-CoV-2); she lived closed to the first Lombardy cluster spreading from Codogno, Italy.

Her clinical, immunological, and genetic patterns have been previously described (as patient no. 4) in a case series of autoimmune polyendocrinopathy-candidiasis-ectodermal dystrophy (APECED), also known as autoimmune polyendocrine syndrome type 1 (APS-1) [2]. In summary, she carries a homozygous R203X mutation in exon five of the autoimmune regulator (AIRE) gene, resulting in primary adrenal

G. Beccuti

guglielmo.beccuti@unito.it

1 Department of Medical Sciences, Division of Endocrinology, Diabetes and Metabolism, University of Turin, Corso Dogliotti 14, 10126 Turin, Italy

2 Department of Medical Sciences and Infectious Diseases, Division of Rheumatology, Foundation IRCCS Polyclinic San Matteo, Pavia, Italy

3 Department of Medical Sciences and Infectious Diseases, Division of Infectious and Tropical Diseases, Foundation IRCCS Polyclinic San Matteo, Pavia, Italy

4 Department of Medical Sciences and Infectious Diseases, Division of General Medicine 1, Foundation IRCCS Polyclinic San Matteo, Pavia, Italy

5 Department of Anesthesia and Intensive Care Unit, Foundation IRCCS Polyclinic San Matteo Pavia, Pavia, Italy

6 Department of Clinical-Surgical, Diagnostic and Pediatric Sciences, University of Pavia, Pavia, Italy insufficiency (PAI), hypoparathyroidism, hypogonadism, ectodermal dystrophy, candidiasis, pernicious anemia, and gastrointestinal dysfunction.

Because her chronic hypoparathyroidism was inadequately controlled by standard treatment alone, the patient had been on hormonal replacement therapy with recombinant human parathyroid hormone (rhPTH) (1-84) since January 2018. After starting rhPTH (1-84) $50 \mu \mathrm{g}$ once daily as a subcutaneous injection, she stopped calcitriol and calcium supplementation, having achieved optimal and stable serum calcium levels. Her treatment was specifically approved by the Italian Medicines Agency (AIFA) before the marketing authorization in Italy, and the patient underwent regular follow-up at the University of Turin (Piedmont, Italy).

On February 19, the patient presented to the emergency department of the Cremona Hospital (Lombardy, Italy) with fever, cough, and dyspnea. High-resolution computed tomography showed multiple and bilateral ground-glass opacities of the lungs. Bronchoalveolar lavage fluid was positive for SARS-CoV-2. After an unsuccessful trial of non-invasive ventilation, her progressive respiratory failure required endotracheal intubation and mechanical ventilation. Therefore, she was transferred to the Intensive Care Unit (ICU) of San Matteo Hospital in Pavia (Lombardy, Italy) on February 22. Pharmacological treatment included empirical antiviral regimens with lopinavir/ritonavir and ribavirin, and prophylaxis with hydroxychloroquine, azithromycin, piperacillin/tazobactam, and trimethoprim-sulfamethoxazole. Hemodynamic support required norepinephrine and dobutamine infusion; intravenous hydrocortisone was subsequently introduced at a dose of $300 \mathrm{mg}$ divided in bolus injections over $24 \mathrm{~h}$. After 6 days, the patient was extubated and started helmet continuous positive airway pressure.

During hospitalization, the intravenous glucocorticoid dose was gradually tapered and eventually switched back to the pre-admission oral regimen.

Unfortunately, the patient's rhPTH treatment was interrupted after admission. Although the summary of product 
characteristics (SmPC) does not include diseases requiring intensive care among reasons for discontinuation, rhPTH has not been studied in this specific clinical setting. Therefore, calcitriol and calcium supplementation was needed again, leading to suboptimal serum calcium concentrations. A few days before discharge, the patient resumed treatment with rhPTH (1-84), with sufficient 25-hydroxy vitamin D stores. Following the instructions in the SmPC, oral calcium supplementation was progressively reduced, and calcitriol supplementation was stopped within 4 days, as the patient achieved adequate serum calcium concentrations.

The patient was discharged on March 27, after 37 days of hospitalization, with complete resolution of symptoms and two negative tests for SARS-CoV-2 at an interval of $24 \mathrm{~h}$. Her last chest X-ray showed bronchiolitis obliterans organizing pneumonia-like features that will need radiological and clinical follow-up.

As of March 27, among 86,498 confirmed COVID-19 patients in Italy, $4.3 \%$ required intensive care (data from the Italian Civil Protection Department website https://www. protezionecivile.it—English version available; last access on May 20). Baseline characteristics and outcomes of 1591 patients infected with SARS-CoV-2 admitted to ICUs in Lombardy have been recently published; median length of stay in ICU was 9 days, and almost all the patients required respiratory support, mainly invasive mechanical ventilation (88\%) [3]. As soon as information is also available on the characteristics of non-ICU hospitalized COVID-19 patients in Italy, it would be interesting to explore whether PAI may worsen COVID-19 outcomes, such as length of hospital stay, incidence of acute respiratory distress syndrome, and the need for CPAP therapy and mechanical ventilation.

The ESE statement notes the occurrence of impaired natural immunity function in PAI, with defective action of neutrophils and natural killer cells, independent of the underlying etiology [4], suggesting that exogenous glucocorticoids play a role in modulating the immune system.

Our case might not be considered a paradigmatic example of PAI; at variance with other forms of autoimmune Addison disease, patients with APS-1 have primary immunodeficiency, which explains the T-cell deficiency-related chronic mucocutaneous candidiasis. This feature has relevant clinical consequences; in fact, a recent prospective study showed that the Finnish APS-1 cohort had an increased mortality from infections (standardized mortality ratio 36; 95\% CI 6.4-110) in comparison to the general population [5].

Similarly, an increased risk of infections has been observed also in other forms of Addison's disease, with autoimmune or genetic basis. However, in these forms, there is no clear demonstration of primary immunodeficiency, and the infection risk in Addison's disease could be linked to the types or doses of glucocorticoid replacement.
A population-based, retrospective, open cohort study in the United Kingdom from 1995 to 2018 showed that the Addison's disease cohort, compared with matched controls, had a higher risk of infections of the lower respiratory [adjusted incidence rate ratio (aIRR) $2.11 ; 95 \% \mathrm{CI}$ 1.64-2.69], urinary (aIRR 1.51; 95\% CI 1.29-1.77), and gastrointestinal (aIRR 3.80; 95\% CI 2.99-4.84) tracts, leading to increased use of antimicrobial agents in the primary care setting [6]. Interestingly, the same study showed no increased risk of infection in patients with untreated congenital adrenal hyperplasia (CAH), but an increased infection risk in patients treated for $\mathrm{CAH}$, suggesting that nonphysiological delivery of glucocorticoid replacement may represent a risk factor for the development of infections [6].

All of these findings are in line with the increased infection-related mortality described in the literature. A population-based, retrospective study on the Swedish population from 1987 to 2001 found that the mortality rate resulting from infections in Addison's disease was five times higher than expected (risk ratio 5.57; 95\% CI 2.04-12.14 in women; risk ratio 6.57; 95\% CI 2.56-15.17 in men) [7]. Another Swedish population-based, retrospective study from 1964 to 2004 reported increased mortality from infections in patients with autoimmune PAI (standardized mortality ratio 5.9; $95 \%$ CI 4.0-8.4) [8]. In Norway, a population-based, retrospective study from 1943 to 2005 reported an increase in mortality from infections associated with Addison's disease [10\% among causes of death (95\% CI 5.1-14.9) vs. $6.0 \%$ in the general population] [9].

The first prospective study investigating causes of death in PAI and secondary adrenal insufficiency (SAI) was based on real-world data from 2034 patients (801 PAI, 1,233 SAI) in the European Adrenal Insufficiency Registry (EU-AIR; ClinicalTrials.gov identifier NCT01661387). The primary objective of EU-AIR is to monitor the safety of long-term treatment with once-daily, modified-release hydrocortisone and other glucocorticoid replacement therapies in adrenal insufficiency. Of the 26 deaths registered from 2012 to 2017 , only $8 \%$ occurred to subjects with Addison's disease. Infections accounted for $15 \%$ of the deaths. With the limitations of a small percentage of autoimmune PAI among infection-related deaths and the EU-AIR inclusion of European adrenal insufficiency patients treated in highly specialized centers, the results of this observational, open-ended study appear to be consistent with the previous evidence from retrospective studies [10].

In conclusion, it is not clear whether the disease per se or the hormonal replacement without physiological glucocorticoid rhythm plays a pre-eminent role in the impaired immune function of PAI. Our case report, along with the aforementioned studies, calls attention to the increased risk of infections in Addison's disease, with or without associated primary immunodeficiency. Further research worldwide 
is required to conclude that a predisposition to COVID19 exists and to assess its adverse short- and long-term outcomes.

Funding This research did not receive any specific grant from any funding agency in the public, commercial, or not-for-profit sector.

\section{Compliance with ethical standards}

Conflict of interest SM received fees for lectures from GE Healthcare, outside the present work. GAI received fees for lectures by Hamilton Medical, Eurosets, Getinge, Intersurgical SpA, Burke \& Burke, outside the present work. FM received fees for lectures from GE Healthcare, Hamilton Medical, SEDA SpA, outside the present work. A research agreement is active between the University of Pavia and Hamilton Medical, outside the present work. The other authors declare that there is no conflict of interest that could be perceived as prejudicing the impartiality of the research reported.

Ethical approval Clinical data were collected in accordance with local ethics requirements for case reports. The study was performed in line with the principles of the Declaration of Helsinki.

Informed consent The patient has consented to the submission of the case report to the journal.

\section{References}

1. Puig-Domingo M, Marazuela M, Giustina A (2020) COVID-19 and endocrine diseases a statement from the European Society of Endocrinology. Endocrine 68:2-5. https://doi.org/10.1007/s1202 0-020-02294-5

2. Betterle C, Ghizzoni L, Cassio A, Baronio F, Cervato S, Garelli S, Barbi E, Tonini G (2012) Autoimmune-polyendocrinopathycandidiasis-ectodermal-dystrophy in Calabria: clinical, immunological and genetic patterns. J Endocrinol Invest 35:877-881. https ://doi.org/10.3275/8109

3. Grasselli G, Zangrillo A, Zanella A, Antonelli M, Cabrini L, Castelli A, Cereda D, Coluccello A, Foti G, Fumagalli R, Iotti G,
Latronico N, Lorini L, Merler S, Natalini G, Piatti A, Ranieri MV, Scandroglio AM, Storti E, Cecconi M, Pesenti A (2020) COVID19 Lombardy ICU network baseline characteristics and outcomes of 1591 patients infected with SARS-CoV-2 admitted to ICUs of the Lombardy Region, Italy. JAMA 323:1574-1581. https://doi. org/10.1001/jama.2020.5394

4. Bancos I, Hazeldine J, Chortis V, Hampson P, Taylor AE, Lord JM, Arlt W (2017) Primary adrenal insufficiency is associated with impaired natural killer cell function: a potential link to increased mortality. Eur J Endocrinol 176:471-480. https://doi. org/10.1530/EJE-16-0969

5. Borchers J, Pukkala E, Mäkitie O, Laakso S (2020) Patients with APECED have increased early mortality due to endocrine causes, malignancies and infections. J Clin Endocrinol Metab 105:140. https://doi.org/10.1210/clinem/dgaa140

6. Tresoldi AS, Sumilo D, Perrins M, Toulis KA, Prete A, Reddy N, Wass JAH, Arlt W, Nirantharakumar K (2020) Increased infection risk in Addison's disease and congenital adrenal hyperplasia. J Clin Endocrinol Metab 105:418-429. https://doi.org/10.1210/ clinem/dgz006

7. Bergthorsdottir R, Leonsson-Zachrisson M, Oden A, Johannsson G (2006) Premature mortality in patients with Addison's disease: a population-based study. J Clini Endocrinol Metab 91:48494853. https://doi.org/10.1210/jc.2006-0076

8. Bensing S, Brandt L, Tabaroj F, Sjoberg O, Nilsson B, Ekbom A, Blomqvist P, Kämpe O (2008) Increased death risk and altered cancer incidence pattern in patients with isolated or combined autoimmune primary adrenocortical insufficiency. Clin Endocrinol (Oxf) 69:697-704. https://doi.org/10.111 1/j.1365-2265.2008.03340.x

9. Erichsen MM, Lovas K, Fougner KJ, Svartberg J, Hauge ER, Bollerslev J, Berg JP, Mella B, Husebye ES (2009) Normal overall mortality rate in Addison's disease, but young patients are at risk of premature death. Eur J Endocrinol 160:233-237. https:// doi.org/10.1530/EJE-08-0550

10. Quinkler M, Ekman B, Zhang P, Isidori AM, Murray RD (2018) EU-AIR investigators mortality data from the European Adrenal Insufficiency Registry-Patient Characterization and Associations. Clin Endocrinol (Oxf) 89:30-35. https://doi.org/10.1111/ cen.13609

Publisher's Note Springer Nature remains neutral with regard to jurisdictional claims in published maps and institutional affiliations. 